\title{
Flap endonuclease 1 silencing is associated with increasing the cisplatin sensitivity of SGC-7901 gastric cancer cells
}

\author{
CHUNHONG XIE $^{1 *}$, KEJIA WANG $^{2 *}$ and DAORONG $\mathrm{CHEN}^{1}$ \\ ${ }^{1}$ Department of Gastroenterology, The First Affiliated Hospital of Chongqing Medical University; \\ ${ }^{2}$ Department of Gastroenterology, Banan People's Hospital of Chongqing, Chongqing 400016, P.R. China
}

Received December 30, 2014; Accepted September 24, 2015

DOI: $10.3892 / \mathrm{mmr} .2015 .4567$

\begin{abstract}
Flap endonuclease 1 (FEN1), which is key in DNA replication and repair, has been demonstrated to be intimately involved in the development and progression of cancer. Our previous study determined that the downregulation of FEN1 can suppress the proliferation of, and induce apoptosis in, gastric cancer SGC-7901 cells. In addition, several FEN1 inhibitors have been identified to increase sensitisation to DNA injury agents. These results may provide a promising treatment method to enhance the traditional chemotherapeutics used for the treatment of gastric cancer. Thus, the aim of the present study was to determine the role of FEN1 in the chemosensitivity of SGC-7901 cells. The protein expression levels of FEN1 in cisplatin (CDDP)-treated SGC-7901 cells were detected using western blot analysis. FEN1 was silenced via specific FEN1-targeted small interfering RNAs (siRNA). The survival and apoptotic rates of the SGC-7901 cells were assessed using an MTT assay and flow cytometry, respectively. Relevant apoptotic factors were detected using western blotting. The results showed that the expression of FEN1 was significantly induced by CDDP in a dose- and time-dependent manner. The targeting of FEN1 in SGC-7901 cells, in combination with CDDP treatment, significantly inhibited their proliferation and effectively increased their apoptotic rate. In addition, in the cells targeted with FEN1-siRNA and exposed to CDDP, the levels of $\mathrm{Bcl}-2$-associated $\mathrm{X}$ protein were significantly increased, whereas the expression levels of Bcl-2 and Bcl-extra large were effectively decreased, compared with the cells exposed to negative control-siRNA and CDDP. These results suggest a potential chemotherapeutic target, which exhibits enhanced sensitivity to CDDP following
\end{abstract}

Correspondence to: Professor Daorong Chen, Department of Gastroenterology, The First Affiliated Hospital of Chongqing Medical University, 1 Youyi Road, Chongqing 400016, P.R. China E-mail: qbhss1903@163.com

${ }^{*}$ Contributed equally

Key words: flap endonuclease 1, small interfering RNA, SGC-7901, cisplatin, sensitivity
FEN1 silencing in SGC-7901 cells via decreased survival and increased apoptosis.

\section{Introduction}

Gastric cancer is one of the major causes of cancer-associated mortality (1) and is the second leading cause of cancer-associated mortality worldwide $(2,3)$, with an overall five-year survival rate of only $20-25 \%$ (4). To date, although surgical resection remains the predominant curative treatment for gastric cancer (5), chemotherapy remains an essential component of the comprehensive treatment of gastric cancer, particularly advanced gastric cancer.

Cisplatin (CDDP), which binds to DNA to generate DNA adducts (6), is a widely used chemotherapeutic agent for the treatment of several types of solid tumour, including gastric cancer. However, its therapeutic efficacy is usually limited by significant toxicity and the resistance of gastric cancer cells to CDDP (7-9). Although the mechanisms underlying the resistance to CDDP are multifactorial, accumulating evidence suggests an important association between drug resistance and DNA repair capability $(10,11)$. As CDDP efficiency is determined by the balance between DNA damage, DNA synthesis inhibition and DNA repair capability (12), there is a requirement for the identification of agents, which can sensitise gastric cancer cells to CDDP by inhibiting different proteins in the DNA repair pathways.

Flap endonuclease 1 (FEN1), a multifunctional and structure-specific nuclease (13), is a key enzyme, which functions in DNA replication and repair to avoid genomic instability (14). It is widely known for its involvement in the penultimate stages of Okazaki fragment maturation and long-patch-base excision repair (LP-BER) (14,15). However, FEN1 has been found to be overexpressed in several types of human cancer (16-19) and cancer cell lines (20-23), indicating that it is a promising diagnostic biomarker in breast, ovarian and gastric cancer $(23,24)$. Thus, abnormal expression of FEN1 may be associated with cancer development and disease progression (25), leading to cancer susceptibility (26). Of note, it has been reported that FEN1 is a useful target for chemotherapeutic development (24). Nikolova et al confirmed that the downregulation of FEN1 in LN308 glioma cells improved their sensitivity to methylating agents, including CDDP, to suppress cell proliferation (19). In additional, our previous study demonstrated that 
downregulation of the expression of FEN1 inhibits proliferation and reduces apoptosis (23). These data suggest that FEN1 may also be an effective therapeutic target in cancer. The efficacy of the alkylating agents can be improved by inhibiting the DNA repair pathways (27), as nucleotide excision repair, in which FEN1 is involved, is pivotal in DNA repair and is associated with resistance to platinum-based chemotherapy (11). Thus, taking into account the fact that FEN1 is a DNA repair protein, the present study aimed to determine whether CDDP can regulate the expression of FEN1, and whether changes to the expression of FEN1 may provide a novel target for enhanced chemotherapeutic results in the treatment of gastric cancer.

To develop a novel chemotherapeutic combination, which increases the sensitivity of SGC-7901 cells to CDDP, the present study investigated the functional significance of FEN1 in CDDP-treated SGC-7901 cells.

\section{Materials and methods}

Human gastric cancer cells (SGC-7901) transfected with FEN1-small interfering (si)RNA (sense 5'-GGACUUGUA GUCCUGCGAUTT-3' and antisense 5'-AUCGCAGGACUA CAAGUCCTT-3') and negative control-siRNA (NC-siRNA) (Invitrogen; Thermo Fisher Scientific, Inc., Waltham, MA, USA) were previously established. FEN1, Bcl-2-associated X protein (Bax), Bcl-2 and Bcl-extra larger (xl) antibodies were purchased from Epitomics (Burlingame, CA, USA). $\beta$-actin antibody was purchased from Boster Bioengineering Co. Ltd. (Wuhan, China). Cisplatin (diluted in RPMI 1640 medium to a final concentration of $1 \mathrm{mM}$ ) was purchased from Sigma-Aldrich (St. Louis, MO, USA) and then maintained at $4^{\circ} \mathrm{C}$ in the dark.

Cell culture. The SGC-7901 human gastric cancer cells, obtained from the Molecular Medicine and Cancer Research Centre of Chongqing Medical University (Chonqing, China) were routinely cultured in RPMI-1640 medium (GE Healthcare Life Sciences, Logan, UT, USA) containing 10\% foetal bovine serum (Invitrogen; Thermo Fisher Scientific, Inc.) and 1\% penicillin-streptomycin (GE Healthcare Life Sciences) at $37^{\circ} \mathrm{C}$ in a $\mathrm{CO}_{2}$ incubator. The cells $\left(3 \times 10^{5}\right.$ per well) were seeded in six-well plates in growth medium, and were cultured overnight to allow adherence. On reaching 60-70\% confluence, the cells were treated with 10, 20, 30, 40 and $50 \mu \mathrm{M}$ CDDP (Sigma-Aldrich) for $24 \mathrm{~h}$ at $37^{\circ} \mathrm{C}$, and with $30 \mu \mathrm{M}$ CDDP for 24,48 and $72 \mathrm{~h}$ at $37^{\circ} \mathrm{C}$. These cells were used to examine the expression levels of FEN1 induced by CDDP using western blotting.

Preparation and transfection of cells with siRNAs. The cells, at a density of $3 \times 10^{5}$ cells per well, were cultured on six-well plates in growth medium, without antibiotics, overnight to allow adherence. On reaching 30-50\% confluence, the cells were transfected using $5 \mu \mathrm{l}$ Lipofectamine ${ }^{\circledR} 2000$ transfection reagent (Invitrogen; Thermo Fisher Scientific, Inc.) diluted in $250 \mu \mathrm{l}$ RPMI-1640 medium, at room temperature. Briefly, $5 \mu \mathrm{l}$ of the recombinant lentiviral vector for the FEN1 gene (FEN1-siRNA) and null vector (NC-siRNA), and the transfection reagent were diluted in $250 \mu$ l RPMI-1640 medium without foetal bovine serum. Following combining of the mixture of siRNA and
Lipofectamine ${ }^{\circledR} 2000$ at room temperature for $20 \mathrm{~min}$, the mixture was added to each well. Following incubation for $6 \mathrm{~h}$, the transfection complexes were removed and replaced with culture medium. Following incubation with CDDP $(30 \mu \mathrm{M})$ in culture medium, the cells were divided into two groups: siRNA-FEN1+CDDP group and NC-siRNA+CDDP group. These cells were used for further experiments.

MTT cell survival assays. The viabilities of the cells were measured using MTT cell survival assays. Briefly, the cells were seeded at a density of $5 \times 10^{3}$ cells per well on 96 -well plates and cultured overnight. At $6 \mathrm{~h}$ post-transfection, the cells in the FEN1-siRNA+CDDP and NC-siRNA+CDDP groups were treated either with increasing concentrations of $\operatorname{CDDP}(0,2.5$, $5,10,20,30,40$ and $50 \mu \mathrm{M})$ for $48 \mathrm{~h}$, or with $10 \mu \mathrm{M}$ CDDP for 24,48 and $72 \mathrm{~h}$ ) to assess the viability of the SGC-7901 cells exposed to CDDP. Subsequently, $20 \mu 1$ MTT (Sigma-Aldrich), which was diluted in phosphate-buffered saline (PBS) to obtain a concentration of $5 \mathrm{mg} / \mathrm{ml}$, was added to each well, and the plates were maintained at $37^{\circ} \mathrm{C}$ for $4 \mathrm{~h}$. The supernatant was then removed, and $150 \mu \mathrm{l}$ dimethyl sulfoxide (Sigma-Aldrich) was added to each well to terminate the reaction. The absorbance was then measured spectrophotometrically at $450 \mathrm{~nm}$ using an Elx800 microplate reader (Bio-Tek Instruments, Inc., Winooski, VT, USA). All the experiments were performed independently at least three times.

Detection of apoptotic cells using flow cytometry. The number of apoptotic cells was analysed using flow cytometry with a fluorescein-isothiocyanate-labelled enhanced Annexin V/Propidium Iodide (PI) Apoptosis Detection kit (Invitrogen; Thermo Fisher Scientific, Inc.). According to the manufacturer's protocol, the adherent and suspended cells were harvested, centrifuged at $4,800 \mathrm{x} g$ for $5 \mathrm{~min}$, washed twice with PBS, and resuspended in $15 \mathrm{ml}$ binding buffer in 0.1 M PBS (Invitrogen; Thermo Fisher Scientific, Inc.). The cells were then subjected to flow cytometry within $1 \mathrm{~h}$. The apoptotic rates were quantified using Cell Quest software (version 3.3; BD Biosciences, San Jose, CA, USA). All the assays were performed three times independently.

Western blot analysis. Western blot analysis was used to verify the inhibitory effects of FEN1-siRNA and to analyse the expression levels of Bax, Bcl-2 and Bcl-xl. Briefly, following the different treatment procedures, the cells were lysed to extract the total protein using Radioimmunoprecipitation Assay Lysis Buffer (Beyotime Institute of Biotechnology, Haimen, China). The concentrations of the extracted proteins were quantified using a Bicinchoninic Acid Protein Assay kit (Beyotime Institute of Biotechnology). The samples were degenerated by boiling in a water bath at $100^{\circ} \mathrm{C}$ for 5 min until fully denatured. The samples ( $40 \mu \mathrm{g}$ protein per lane) and pre-stained molecular weight markers were separated by $10 \%$ SDS-PAGE and transferred electrophoretically onto polyvinylidene fluoride membranes in a minigel apparatus (Mini-PROTEAN II; Bio-Rad Laboratories, Inc., Hercules, CA, USA). The membranes were blocked using 5\% skimmed-milk powder in Tris-buffered saline with Tween 20 (TBST; Beyotime Institute of Biotechnology), containing $10 \mathrm{mM}$ Tris- $\mathrm{HCl}$ ( $\mathrm{pH} 7.5)$, $150 \mathrm{mM} \mathrm{NaCl}$ and $0.1 \%$ Tween-20 for $1 \mathrm{~h}$ at room temperature. The membranes were washed with three times with TBST for 

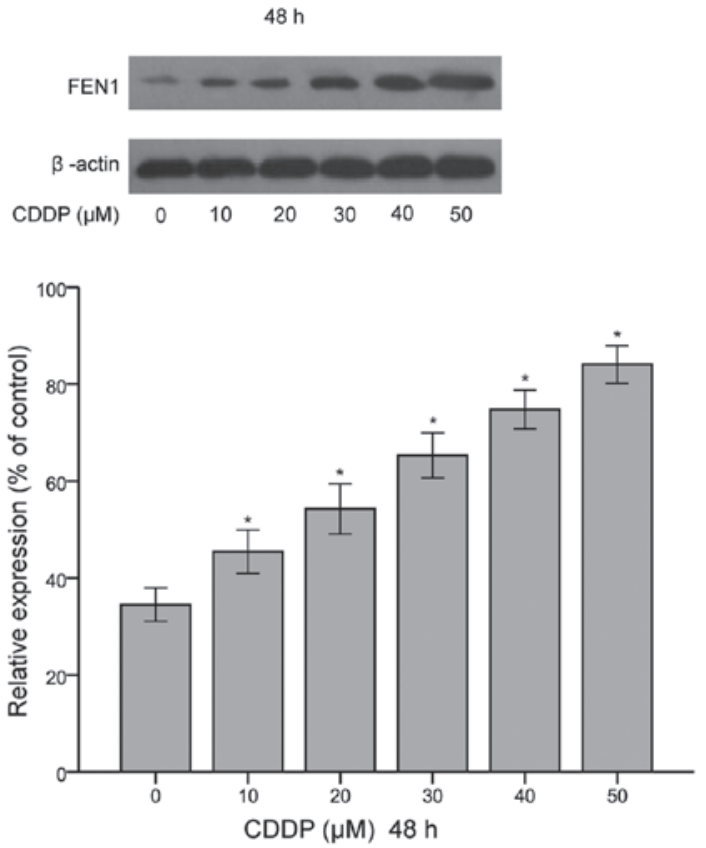

B
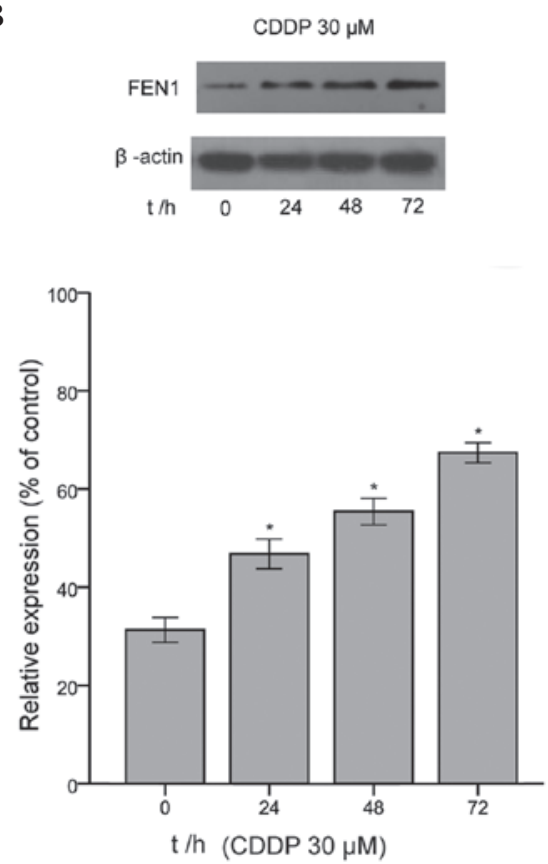

Figure 1. Expression of FEN1 is upregulated in CDDP-treated SGC-7901 cells. (A) Following treatment with CDDP (10, $20,30,40$, and 50 mM for 48 h and $30 \mu \mathrm{M}$ CDDP for 24, 48 and $72 \mathrm{~h}$ ) the expression of FEN1 was significantly induced by CDDP in a dose- and time-dependent manner, compared with the untreated control group. The protein levels were determined using western blotting, and representative images are shown. (B) Protein levels were quantified using densitometry. The results are presented as the mean \pm standard deviation of three independent experiments, standardized to $\beta$-actin and normalised to $100 \%$. The expression of FEN1 was significantly increased in the CDDP-treated group, compared with the control group * $<0.05$, vs. control. CDDP, cisplatin; FEN1, flap endonuclease $1 ; \mathrm{t} / \mathrm{h}$, time (h).

$10 \mathrm{~min}$. The membranes were then incubated with antibodies against rabbit monoclonal FEN1 (1:1,000; cat. no. ab133311; Epitomics; Abcam, Cambridge, MA, USA), rabbit monoclonal Bax (1:1,000; cat. no. ab32503; Epitomics; Abcam), rabbit monoclonal Bcl-2 (1:1,000; cat. no. ab32124; Epitomics; Abcam), rabbit monoclonal Bcl-xl (1:1,000; cat. no. ab32370; Epitomics; Abcam), and rabbit polyclonal $\beta$-actin $(1: 1,000$; cat. no. BA2305; Boster Bioengineering Co., Ltd.) overnight at $4^{\circ} \mathrm{C}$. The following day, the membranes were washed three times with TBST for $30 \mathrm{~min}$ and incubated with horseradish peroxidase-conjugated goat anti-rabbit secondary antibodies (1:1,000; cat. no. BA1055; Boster Bioengineering Co., Ltd.) at room temperature for $2 \mathrm{~h}$. The blots were then washed, incubated with Solution A and Solution B from the BeyoECL Plus western blotting detection reagent kit (cat. no. P0018; Beyotime Institute of Biotechnology) with a Solution A:Solution $B$ ratio of $1: 1$ at room temperature for $10 \mathrm{~min}$, and measured using a chemiluminescence western blotting detection system (Bio-Rad Laboratories, Inc.). All the experiments were performed three times.

Statistical analysis. The quantitative data are reported as the mean \pm standard deviation. All data were analysed using a paired test using IBM SPSS 19.0 software (IBM SPSS, Armonk, NY, USA). All P-values were two-sided and $\mathrm{P}<0.05$ was considered to indicate a statistically significant difference.

\section{Results}

Expression of FEN1 is upregulated in CDDP-treated SGC-7901 cells. Our previous study confirmed that FEN1 is

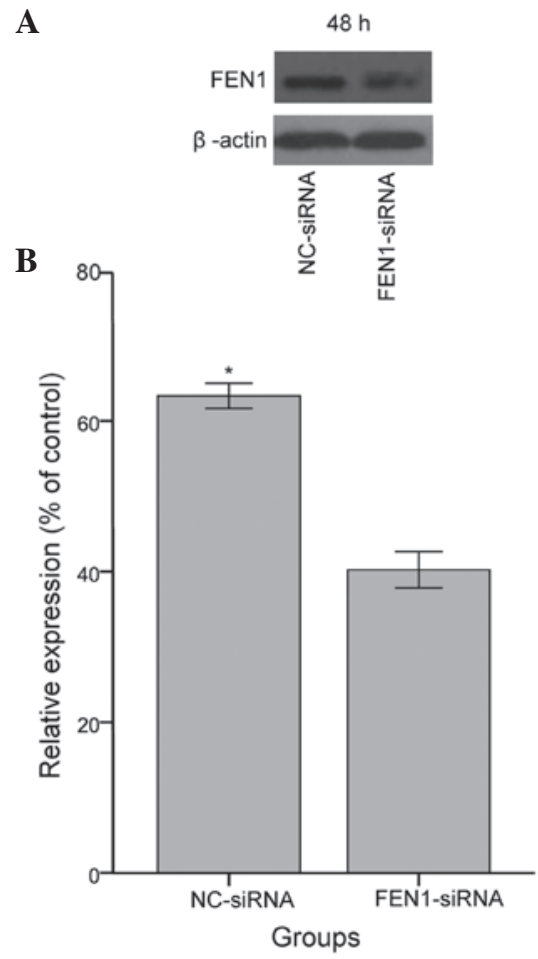

Figure 2. Expression of FEN1 is significantly inhibited by FEN1-siRNA in the SGC-7901 cell line. The SGC-7901 cells were transfected with FEN1-siRNA and NC-siRNA. (A) Expression levels of FEN1 were measured using western blotting $48 \mathrm{~h}$ post-transfection, and representative images are shown. (B) Protein levels were quantified using densitometry. The results are presented as the mean \pm standard deviation of three independent experiments, standardized to $\beta$-actin and normalised to $100 \%$. The expression of FEN1 was significantly decreased in the FEN1-siRNA group, compared with the NC-siRNA group. ${ }^{*} \mathrm{P}<0.05$, vs. NC-siRNA group. FEN1, flap endonuclease 1; $\mathrm{NC}$, normal control; siRNA; small interfering RNA. 
A

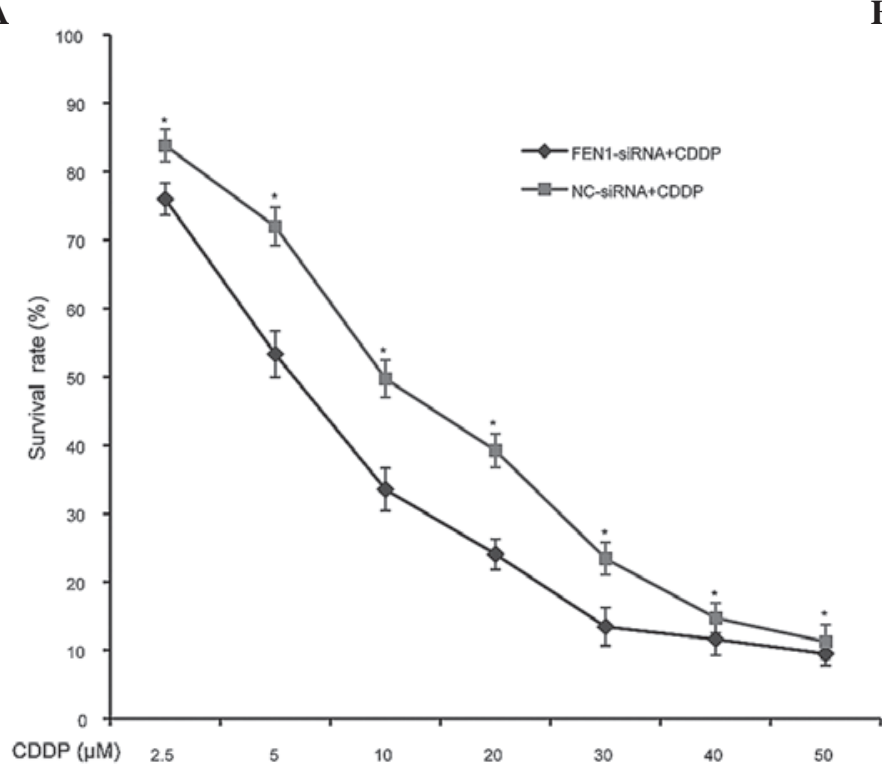

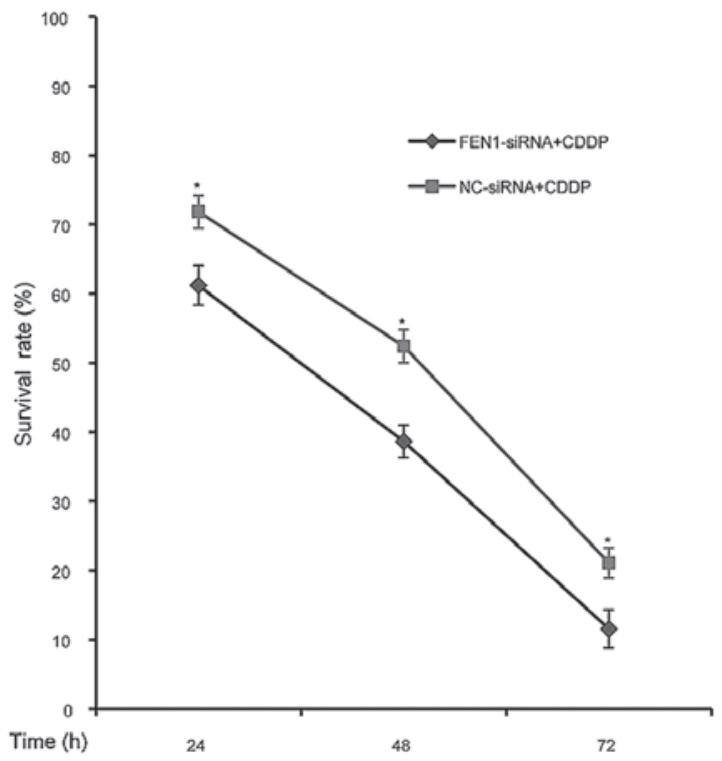

Figure 3. FEN1 silencing enhances the survival rate of SGC-7901 cells. (A) FEN1-siRNA cells and NC-siRNA cells were treated with 0, 10, 20, 30, 40 and $50 \mu \mathrm{M}$ CDDP for $48 \mathrm{~h}$. (B) FEN1-siRNA cells and NC-siRNA cells were treated with $30 \mu \mathrm{M}$ CDDP for 24,48 and $72 \mathrm{~h}$. The survival rates following treatment were detected using an MTT assay. The results are presented as the mean \pm standard deviation of triplicate wells and are representative of at least three independent experiments. The survival rates of the FEN1-siRNA+CDDP cells were significantly lower than those of the NC-siRNA+CDDP cells in all the conditions assessed ( $\mathrm{P}<0.05)$. CDDP, cisplatin; FEN1, flap endonuclease 1; siRNA, small interfering RNA; NC, normal control.

overexpressed in gastric cancer tissues and gastric cell lines, particularly SGC-7901 cells (23). Additionally, it has been found that the expression level of FEN1 is increased in 5-FU-R cells, compared with HCT-116 cells (28), which indicates that the effect of the upregulation of FEN1 may be in response to DNA damage. Thus, in further investigating whether the expression of FEN1 is induced by CDDP in SGC-7901 cells, the present study found that the protein levels of FEN1 were significantly enhanced at a range of CDDP concentrations (10, 20, 30, 40 and $50 \mu \mathrm{M}$ CDDP) for $48 \mathrm{~h}$, and following treatment with $30 \mu \mathrm{M}$ CDDP for 24,48 and $72 \mathrm{~h}$ (Fig. 1). These data revealed that the expression of FEN1 in SGC-7901 cells was also upregulated by exposure to the chemotherapeutic agent, CDDP. This may be explained by the fact the enhanced concentration of CDDP or extended treatment duration resulting in increased DNA damage; however, the increased expression level of FEN1 may have been in response to increased DNA damage.

Expression of FEN1 is markedly inhibited by siRNA in $S G C-7901$ gastric cancer cells. Several FEN1 inhibitors have been reported to permit sensitisation to DNA injury agents $(29,30)$. Our previous study confirmed that FEN1 is upregulated in SGC-7901 cells, and that the levels of FEN1 can be effectively inhibited by FEN1-siRNA (22). Thus, to determine whether FEN1 is involved in sensitivity to CDDP, the present study knocked down FEN1 via the same specific siRNA in SGC-7901 cells and used western blot analysis to verify the inhibitory effects of FEN1-siRNA. As expected, a reduction in the expression of FEN1 was observed in the SGC-7901 cells transfected with FEN1-siRNA, compared with the cells transfected with NC-siRNA, as shown in Fig. $2(\mathrm{P}<0.05)$. In the present study, $\sim 63 \%$ silencing of FEN1 was obtained, relative to the SGC-7901 cells transfected with NC-siRNA. These observations confirmed the suppression of FEN1 protein via specific
FEN1-targeted siRNA, indicating that these cells were suitable for use in further experiments.

FEN1 silencing decreases the survival rate of SGC-7901 cells following CDDP treatment. As FEN1 was overexpressed in SGC-7901 cells and was further upregulated in response to CDDP treatment, the present study hypothesized that the targeting of FEN1 can sensitise SGC-7901 cells to CDDP. Thus, to further determine whether the enhanced sensitivity observed in the FEN1-knockdown SGC-7901 cells following CDDP treatment is reflected at the level of cell survival, the present study performed an MTT assay. As expected, a significantly lower survival rate was observed in the FEN1-siRNA group, compared with the NC-siRNA group following treatment with different concentrations of $\operatorname{CDDP}(0,2.5,5,10,20,30,40$ and $50 \mu \mathrm{M})$, and following treatment for 24,48 and $72 \mathrm{~h}(\mathrm{P}<0.05$; Fig. 3). These results confirmed that the silencing of FEN1 led to reduced survival of the SGC-7901 cells following CDDP treatment, which indicated that FEN1-siRNA transfection effectively increased the sensitivity of the cells to CDDP toxicity.

FEN1 silencing enhances SGC-7901 cell apoptosis induced by $C D D P$. To elucidate the mechanism underlying the sensitivity of FEN1-siRNA cells to CDDP, flow cytometric analysis was performed with Annexin V/propidium iodide apoptosis detection. The early apoptotic (Annexin $\mathrm{V}^{+} / \mathrm{PI}^{-}$) and late apoptotic (Annexin $\mathrm{V}^{+} / \mathrm{PI}^{+}$) cells were included. CDDP induced apoptosis in the NC-siRNA+CDDP cells $(49.74 \pm 4.68 \%)$. However, the apoptotic effect in the FEN1-siRNA+CDDP cells was more marked, with an apoptotic rate of 73.98 $\pm 5.19 \%(\mathrm{P}<0.05$, compared with the NC-siRNA+CDDP group), as shown in Fig. 4. These results indicated that the silencing of FEN1 led to enhanced levels of apoptosis in the SGC-7901 cells following CDDP treatment. 
A
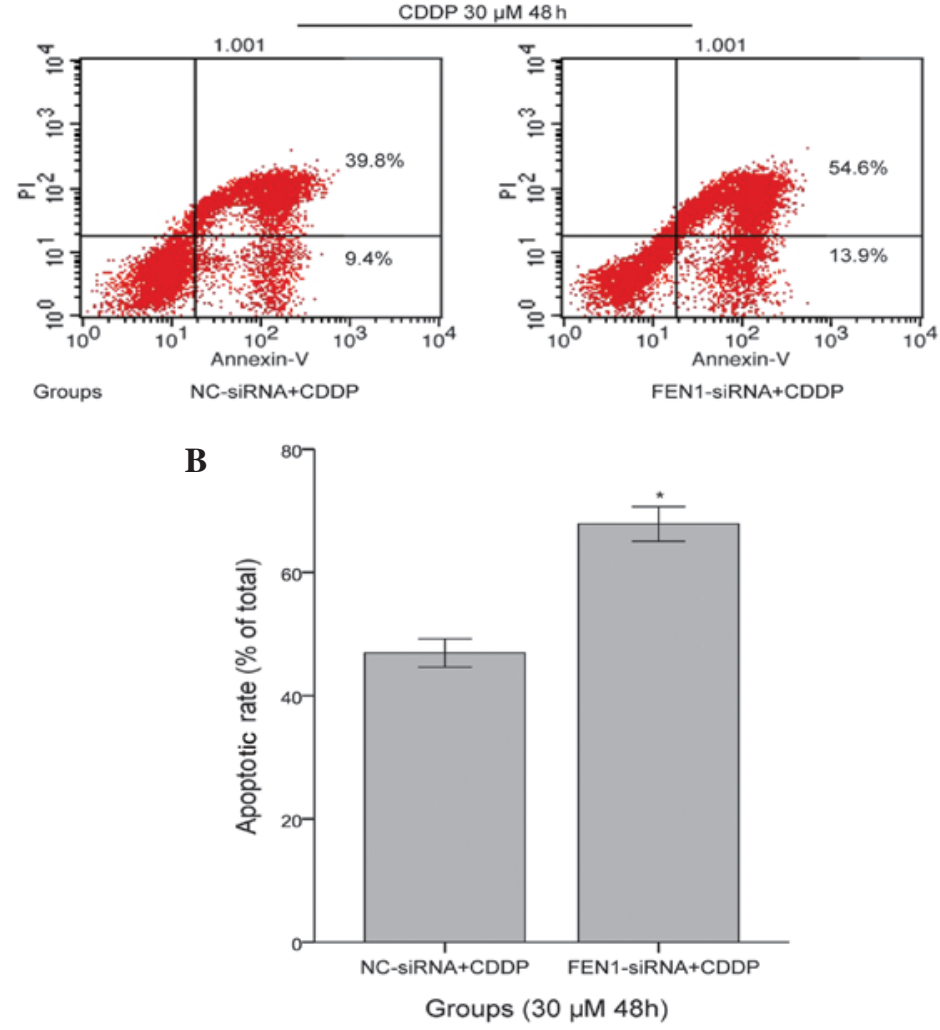

Figure 4. Silencing of FEN1 enhances CDDP-induced apoptosis of SGC-7901 cells. (A) Apoptosis of the two groups of SGC-7901 cells. The cells were assessed using flow cytomettic analysis of Annexin V/propidium iodide. The results include the viable and non-viable apoptotic cells. The apoptotic rates of the FEN1-siRNA+CDDP and NC-siRNA+CDDP cells were quantified using Cell Quest software. Upper left, debris and damaged cells; lower left, negative control normal cells; upper right, early apoptotic cells; lower right, late apoptotic and dead cells. (B) Apoptosis rates of the FEN1-siRNA+CDDP cells were significantly increased, compared with those of the NC-siRNA+CDDP cells ( $\left.{ }^{*} \mathrm{P}<0.05\right)$. The results are expressed as the mean \pm standard deviation and are representative of the results from three independent experiments. CDDP, cisplatin; FEN1, flap endonuclease 1; siRNA, small interfering RNA; NC, normal control; PI, propidium iodide.

A

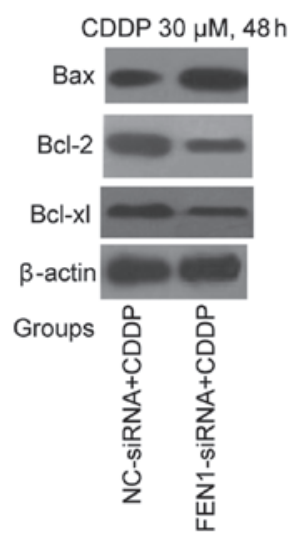

B

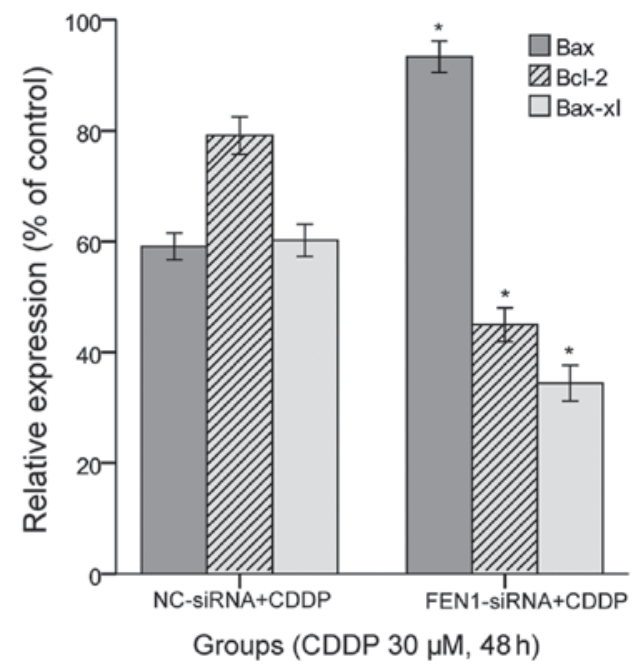

Figure 5. Expression levels of Bcl-2, Bcl-xl and Bax following FEN1 silencing. (A) Expression levels of Bcl-2, Bcl-xl and Bax in the FEN1-siRNA+CDDP and NC-siRNA+CDDP cells were determined using western blotting. Representative images are shown. (B) Protein levels were quantified using densitometry. The results are presented as the mean \pm standard deviation from three independent experiments, standardized to $\beta$-actin and normalised to $100 \%$. The expression of Bax was significantly increased in the FEN1-siRNA+CDDP cells, compared with the NC-siRNA+CDDP cells, whereas the expression levels of Bcl-2 and Bcl-xl were significantly decreased in the FEN1-siRNA+CDDP cells (" $\mathrm{P}<0.05)$, compared with the NC-siRNA+CDDP cells.. CDDP, cisplatin; FEN1, flap endonuclease 1; siRNA, small interfering RNA; NC, normal control; Bcl-2, B cell lymphoma-2; BCL-xl, Bcl-extra large; Bax, Bcl-2-associated X protein.

FEN1 silencing decreases the protein expression levels of $B c l-2$ and $B c l-x l$, and increases the protein expression of Bax. It is well-known that the Bcl-2 family of proteins are integral in regulating apoptosis. Thus, to determine whether the knockdown of FEN1 in SGC-7901 cells combined with CDDP treatment affected the expression of the Bcl-2 family proteins, 
the present study examined the anti-apoptotic family members, Bcl-2 and Bcl-xl, and the pro-apoptotic family member, Bax, via western blotting. As shown in Fig. 5, the expression of Bax was elevated in the FEN1-siRNA+CDDP cells, compared with the NC-siRNA + CDDP cells $(\mathrm{P}<0.05)$. By contrast, the protein expression levels of anti-apoptotic $\mathrm{Bcl}-2$ and $\mathrm{Bcl}-\mathrm{xl}$ were significantly decreased in the FEN1-siRNA+CDDP SGC-7901 cells, compared with the NC-siRNA+CDDP cells (Fig. 5). These relevant apoptotic factors further suggested that the downregulation of FEN1 may enhance apoptosis by increasing the sensitivity to CDDP, and this increase in sensitivity may be associated with the Bcl-2 family proteins, on which further relevant investigations are required.

\section{Discussion}

FEN1, which is involved in RNA primer removal and LP-BER $(14,15)$, interacts with different proteins to execute its function in different pathways to maintain genomic stability $(29,31)$, particularly in DNA replication, DNA repair, maintenance of telomere stability and apoptotic fragmentation of DNA (30). Accumulating evidence has shown that certain FEN1 inhibitors can permit sensitisation to DNA injury agents $(32,33)$, although the mechanism remains to be fully elucidated. CDDP, a well-known DNA-damaging agent, binds to DNA to generate DNA adducts (6), which are repaired in cells primarily through the nucleotide excision repair (NER) pathway (34). It is known that the resistance of cancer cells to CDDP remains a challenging problem, and limits its use in clinical treatment (35). Therefore, a novel and promising concept is the enhancement of the sensitivity of cancer cells to improve the therapeutic efficacy of CDDP. In addition, further evidence also indicates that inhibiting the amino acid Asp181 of FEN1, which affects its endonuclease activity, in combination with treatment with temozolomide, a DNA-alkylating agent, may be an effective strategy (24). Thus, the present study investigated whether alterations in the expression of FEN1 are associated with CDDP treatment.

The present study demonstrated that the FEN1 protein was markedly induced by CDDP in a concentration- and time-dependent manner in SGC-7901 cells, compared with the untreated control cells, indicating that increased expression of FEN1 may lead to the increased DNA repair capability of cells in response to DNA damage. In addition, a previous study reported increased expression of FEN1 in 5-FU-R cells, compared with HCT-116 cells (28), which was in accordance with our findings. As increases in the levels of FEN1 can be induced by CDDP, the present study subsequently examined whether downregulation in the expression of FEN1 to decrease the level of DNA repair enhances the sensitivity to CDDP. Our previous study confirmed that the expression of FEN1 is upregulated in SGC-7901 cells. Thus, FEN1 was silenced in SGC-7901 cells via specific FEN1-targeted siRNA to assess the inhibitory effects of FEN1-siRNA, as shown in Fig. 2. The results revealed that the siRNA-FEN1 cells treated with different concentrations of CDDP for different durations presented with markedly reduced survival rates, compared with the NC-siRNA group, determined using MTT cell survival assays. This finding is consistent with the fact that cells exhibiting deficient DNA repair are markedly more sensitive to CDDP, compared with cells proficient in repair (36). These data suggested that FEN1 silencing may enhance the sensitivity of the cells to CDDP, which may provide a novel and promising strategy for enhancing the effects of chemotherapy. During the development of gastric cancer, cells are subjected to clonal proliferation and apoptosis, and elevated apoptosis provides a potential method for inhibiting tumour survival (37). Our previous study confirmed that the knockdown of FEN1 can induce the apoptosis of SGC-7901 cells (23). In the present study, whether the silencing of FEN1 promotes apoptosis following treatment with CDDP was determined. Notably, the results indicated that the reduced survival observed in the FEN1-siRNA+CDDP group was caused by an increase in the rate of apoptosis, as determined using flow cytometry. These results demonstrated that the knockdown of FEN1 significantly sensitised the SGC-7901 cells to CDDP-induced apoptosis. Taken together, these results indicate that the downregulation of FEN1 may be a novel target for increasing the sensitivity of SGC-7901 cells to CDDP to overcome resistance. A similar finding was obtained in glioblastoma cell lines depleted in FEN1, which showed increased damage sensitivity to CDDP (19).

It is well known that the use of alkylating agents as chemotherapeutic drugs is based on their ability to trigger an apoptotic response (38). In addition, Larsen et al confirmed that two domain-specific FEN1 proteins cause early onset lymphoma and extensive embryonic apoptosis (39). Evidently, a decrease in FEN1 activity may cause abnormal cell proliferation, genomic instability and tumourigenesis. However, the pro-apoptotic mechanism of FEN1 remains to be fully elucidated. In the present study, to investigate the pro-apoptotic mechanism of FEN1, western blotting was performed to analyse the expression levels of anti-apoptotic Bcl-2 and Bcl-xl, and pro-apoptotic Bax. The pro-apoptotic gene, Bax, was overexpressed in the FEN1-siRNA+CDDP group, whereas the anti-apoptotic genes, $\mathrm{Bcl}-2$ and $\mathrm{Bcl}-\mathrm{xl}$, were decreased, compared with the levels observed in the NC-siRNA+CDDP group. To the best of our knowledge, the present study provides the first demonstration that FEN1 is potentially involved in CDDP-induced apoptosis, although the detailed mechanism underlying the action and involvement of other components in the pro-apoptotic effect of FEN1 remain to be elucidated. The mechanism of FEN1 may involve the formation of a complex of FEN1 with other proteins to execute its function through different pathways, and further investigations to determine the possible apoptotic mechanisms involved in targeting FEN1 may provide further insights into this possibility.

In conclusion, the results of the present study revealed that the silencing of FEN1 in SGC-7901 cells enhanced their sensitivity to CDDP. These results indicated that FEN1 can be upregulated by CDDP, and that knockdown of the expression of FEN1 may offer a potential therapeutic approach for enhancing sensitivity to CDDP-based treatment, via decreased survival rate and increased apoptosis of cells. Increased apoptosis was further confirmed by relevant apoptotic factors. These findings not only increase current knowledge on the biology of FEN1, but also present a potential novel strategy for enhancing sensitivity to CDDP, via specific FEN1-targeted siRNA, to inhibit DNA repair. 


\section{Acknowledgements}

The authors would like to thank the Molecular Medicine and Cancer Research Center of Chongqing Medical University for their assistance. This study was supported by the Research Fund of Chongqing Municipal Health Bureau (grant. no. 2009-2-345).

\section{References}

1. Nagini S: Carcinoma of the stomach: A review of epidemiology, pathogenesis, molecular genetics and chemoprevention. World J Gastrointest Oncol 4: 156-169, 2012.

2. Kamangar F, Dores GM and Anderson WF: Patterns of cancer incidence, mortality and prevalence across five continents: Defining priorities to reduce cancer disparities in different geographic regions of the world. J Clin Oncol 24: 2137-2150, 2006

3. Ferlay J, Parkin DM and Steliarova-Foucher E: Estimates of cancer incidence and mortality in Europe in 2008. Eur J Cancer 46 : 765-781, 2010.

4. Meyer HJ and Wilke $\mathrm{H}$ : Treatment strategies in gastric cancer. Dtsch Arztebl Int 108: 698-705, 2011.

5. Chang WJ, Du Y, Zhao X, Ma LY and Cao GW: Inflammation-related factors predicting prognosis of gastric cancer. World J Gastroenterol 20: 4586-4596, 2014.

6. Jamieson ER and Lippard SJ: Structure, recognition and processing of cisplatin-DNA adducts. Chem Rev 99: 2467-2498, 1999.

7. Loehrer PJ and Einhorn LH: Drugs five years later. Cisplatin. Ann Intern Med 100: 704-713, 1984.

8. Reedijk J: New clues for platinum antitumor chemistry: Kinetically controlled metal binding to DNA. Proc Natl Acad Sci USA 100: 3611-3616, 2003.

9. Woźniak K and Błasiak J: Recognition and repair of DNA-cisplatin adducts. Acta Biochim Pol 49: 583-596, 2002.

10. Olaussen KA, Dunant A, Fouret P, Brambilla E, André F, Haddad V, Taranchon E, Filipits M, Pirker R, Popper HH, et al: DNA repair by ERCC1 in non-small-cell lung cancer and cisplatin-based adjuvant chemotherapy. N Engl J Med 355 983-991, 2006.

11. Reed E: Platinum-DNA adduct, nucleotide excision repair and platinum based anti-cancer chemotherapy. Cancer Treat Rev 24 331-344, 1998

12. Xu Y, Wang C and Li Z: A new strategy of promoting cisplatin chemotherapeutic efficiency by targeting endoplasmic reticulum stress. Mol Clin Oncol 2: 3-7, 2014.

13. Harrington JJ and Lieber MR: The characterization of amammalian DNA structure-specific endonuclease. EMBO J 13: $1235-1246,1994$

14. Henneke G, Friedrich-Heineken E and Hübscher U: Flap endo-nuclease 1: A novel tumour suppressor protein. Trends Biochem Sci 28: 384-390, 2003.

15. Klungland A and Lindahl T: Second pathway for completion of human DNA base excision-repair: Reconstitution with purifiedproteins and requirement for DNase IV (FEN1). EMBO J 16 3341-3348, 1997.

16. Lam JS, Seligson DB, Yu H, Li A, Eeva M, Pantuck AJ, Zeng G, Horvath S and Belldegrun AS: Flap endonuclease 1 is overex-pressed in prostate cancer and is associated with a high Gleason score. BJU Int 98: 445-451, 2006.

17. Singh P, Yang M, Dai H, Yu D, Huang Q, Tan W, Kernstine KH, Lin D and Shen B: Overexpression and hypomethylation of flap endonuclease 1 gene in breast and other cancers. Mol Cancer Res 6: 1710-1717, 2008.

18. Iacobuzio-Donahue CA, Maitra A, Olsen M, Lowe AW, van Heek NT, Rosty C, Walter K, Sato N, Parker A, Ashfaq R, et al: Exploration of global gene expression patterns in pancreatic adenocarcinoma using cDNA microarrays. Am J Pathol 162 $1151-1162,2003$

19. Nikolova T, Christmann M and Kaina B: FEN1 is overexpressed in testis, lung and brain tumors. Anticancer Res 29: 2453-2459, 2009.
20. Sato M, Girard L, Sekine I, Sunaga N, Ramirez RD, Kamibayashi C and Minna JD: Increased expression and no mutation of the Flap endonuclease (FEN1) gene in human lung cancer. Oncogene 22: 7243-7246, 2003.

21. LaTulippe E, Satagopan J, Smith A, Scher H, Scardino P, Reuter V and Gerald WL: Comprehensive gene expression analysis of prostate cancer reveals distinct transcriptional programs associated with metastatic disease. Cancer Res 62: 4499-4506, 2002.

22. Wang K, Xie C and Chen D: Flap endonuclease 1 is a promising candidate biomarker in gastric cancer and is involved in cell proliferation and apoptosis. Int J Mol Med 33: 1268-1274, 2014

23. Abdel-Fatah TM, Russell R, Albarakati N, Maloney DJ, Dorjsuren D, Rueda OM, Moseley P, Mohan V, Sun H, Abbotts R, et al: Genomic and protein expression analysis reveals flap endnuclease 1 (FEN1) as a key biomarker in breast and ovarian cancer. Mol Oncol 87: 1326-1338, 2014.

24. Panda H, Jaiswal AS, Corsino PE, Armas ML, Law BK and Narayan S: Amino acid Asp181 of 5'-flap endonuclease 1 is a useful target for chemotherapeutic development. Biochemistry 48 : 9952-9958, 2009.

25. Zheng L, Dai H, Zhou M, Li M, Singh P, Qiu J, Tsark W, Huang Q, Kernstine $\mathrm{K}$, Zhang X, et al: FEN1 mutations result in autoimmunity, chronic inflammation and cancers. Nat Med 13: 812-819, 2007.

26. Liu L, Zhou C, Zhou L, Peng L, Li D, Zhang X, Zhou M, Kuang P, Yuan Q, Song X and Yang M: Functional FEN1 genetic variants contribute to risk of hepatocellular carcinoma, esophageal cancer, gastric cancer and colorectal cancer. Carcinogenesis 33: 119-123, 2012.

27. Middleton MR and Margison GP: Improvement of chemotherapy efficacy by inactivation of a DNA-repair pathway. Lancet Oncol 4 : 37-44, 2003.

28. Das D, Preet R, Mohapatra P, Satapathy SR and Kundu CN: 1,3-Bis (2-chloroethyl)-1-nitrosourea enhances the inhibitory effect of resveratrol on 5-fluorouracil sensitive/resistant colon cancer cells. World J Gastroenterol 19: 7374-7388, 2013.

29. Dianova II, Bohr VA and Dianov GL: Interaction of human AP endonuclease 1 with flap endonuclease 1 and proliferating cell nuclear antigen involved in long-patch base excision repair. Biochemistry 40: 12639-12644, 2001

30. Saharia A, Guittat L, Crocker S, Lim A, Steffen M, Kulkarni S and Stewart SA: Flap endonuclease 1 contributes to telomere stability. Curr Biol 18: 496-500, 2008.

31. Parrish JZ, Yang C, Shen B and Xue D: CRN-1, a Caenorhabditis elegans FEN1 homologue, cooperates with CPS-6/EndoG to promote apoptotic DNA degradation. EMBO J 22: 3451-3460, 2003.

32. Tumey LN, Huck B, Gleason E, Wang J, Silver D, Brunden K, Boozer S, Rundlett S, Sherf B, Murphy S, et al: The identification and optimization of 2,4-diketobutyric acids as flap endonuclease 1 inhibitors. Bioorg Med Chem Lett 14: 4915-4918, 2004

33. Tumey LN, Bom D, Huck B, Gleason E, Wang J, Silver D, Brunden K, Boozer S, Rundlett S, Sherf B, et al: The identification and opti-mization of a N-hydroxy urea series of flap endonuclease 1 inhibitors. Bioorg Med Chem Lett 15: 277-281, 2005.

34. Chu G: Cellular responses to cisplatin. The roles of DNA-binding proteins and DNA repair. J Biol Chem 269: 787-790, 1994.

35. Galluzzi L, Vitale I, Michels J, Brenner C, Szabadkai G, Harel-Bellan A, Castedo M and Kroemer G: Systems biology of cisplatin resistance: Past, present and future. Cell Death Dis 5: e1257, 2014.

36. Popoff SC, Beck DJ and Rupp WD: Repair of plasmid DNA damaged in vitro with cis- or trans-diamminedichloroplatinum(II) in Escherichia coli. Mutat Res 183: 129-137, 1987.

37. Fuertes MA, Alonso C and Pérez JM: Biochemical modulation of cisplatin mechanisms of action: Enhancement of antitumor activity and circumvention of drug resistance. Chem Rev 103: 645-662, 2003

38. Sawyers C: Targeted cancer therapy. Nature 432: 294-297, 2004.

39. Larsen E, Kleppa L, Meza TJ, Meza-Zepeda LA, Rada C, Castellanos CG, Lien GF, Nesse GJ, Neuberger MS, Laerdahl JK, et al: Early-onset lymphoma and extensive embryonic apoptosis in two domain-specific Fen1 mice mutants. Cancer Res 68: 4571-4579, 2008. 